\title{
BMJ Open Effectiveness of nicotine replacement therapy sample at outdoor smoking hotspots for initiating quit attempts and use of smoking cessation services: a protocol for a cluster randomised controlled trial
}

Yee Tak Derek Cheung (D , , ${ }^{1}$ Ching Han Helen Chan, ${ }^{2}$ Kin Sang Ho, ${ }^{2}$ Celeste Tang, ${ }^{2}$ Chloe Wing Hei Lau, ${ }^{1}$ William Ho Cheung Li (D) , ${ }^{1}$ Man Ping Wang (D) , 1 Tai Hing Lam ${ }^{3}$

To cite: Cheung YTD, Chan $\mathrm{CHH}$, Ho KS, et al. Effectiveness of nicotine replacement therapy sample at outdoor smoking hotspots for initiating quit attempts and use of smoking cessation services: a protocol for a cluster randomised controlled trial. BMJ Open 2020;10:e036339. doi:10.1136/ bmjopen-2019-036339

- Prepublication history for this paper is available online. To view these files, please visit the journal online (http://dx.doi. org/10.1136/bmjopen-2019036339).

Received 11 December 2019 Revised 09 March 2020 Accepted 12 March 2020
Check for updates

(C) Author(s) (or their employer(s)) 2020. Re-use permitted under CC BY-NC. No commercial re-use. See rights and permissions. Published by BMJ.

For numbered affiliations see end of article.

Correspondence to Dr Yee Tak Derek Cheung; derekcheung@hku.hk

\section{ABSTRACT}

Introduction More than half of the smoking population in Hong Kong are unmotivated to quit. Only about $2 \%$ of tobacco users in the territory have ever used cessation aids such as nicotine replacement therapy (NRT). The present study aims to assess the effectiveness of delivering 1-week free NRT sample plus brief intervention to smokers at outdoor smoking hotspots on quit attempts and use of smoking cessation services.

Methods and analysis This is a two-arm, pragmatic, multisite, cluster randomised controlled trial (RCT) on the effectiveness of increasing quit attempts, use of cessation service and recruitment outcomes. Trained smoking cessation ambassadors will approach smokers at outdoor smoking hotspots, and deliver brief smoking cessation advice. Recruitment sessions are randomised to intervention or control group (allocation ratio 1:1). Participants in the intervention group $(n=550)$ will receive 1-week free NRT sample (either patch or gum), brief medication advice from an onsite nurse and cessation service referral, whereas participants in control group $(n=275)$ will only receive the brief advice and service referral. The primary outcomes are the proportion of participants who enrol in any cessation service in Hong Kong within 1 month of the recruitment, and the proportion of participants who report quit attempts at 1-month follow-up. Secondary outcomes include self-reported use of NRT, self-reported 7-day tobacco abstinence, 30-day abstinence at 3 months and 6 months, biochemically validated abstinence at 6 months, perceived importance, difficulty and confidence to quit (scale 0-10), and Incremental Behavior Change towards Smoking Cessation. Process outcomes include number of smokers who will be approached, will accept the brief smoking cessation advice or be recruited to participate in the RCT.

Ethics and dissemination The Institutional Review Board of the University of Hong Kong/Hospital Authority Hong Kong West Cluster approved the trial (UW 18-118). Findings will be disseminated through funding website, publication and conference presentations.
Strengths and limitations of this study

- This is a large trial to investigate the effectiveness of delivering sample of nicotine replacement therapy (NRT) at outdoor smoking hotspots for recruitment of smokers and quitting.

- Complete NRT compliance is not mandatory.

- Cluster randomisation prior to recruitment cannot conceal trial group allocation.

- Consent, baseline assessment and intervention delivery are carried out flexibly to enhance the recruitment and smokers' interest.

Trial registration number NCT03717051

\section{INTRODUCTION}

Smoking causes cancers, coronary heart diseases and many more chronic diseases. Worldwide, about 933.1 million are daily smokers, ${ }^{1}$ and half of them will die of smoking-related diseases. ${ }^{2}$ Cost-covered cessation services and medications are recommended by WHO as effective strategies, ${ }^{3}$ but the utilisation level of these cessation aids is very low. $^{45}$

Hong Kong's daily smoking prevalence is the lowest (10.0\% in 2017) in the developed world, but less than half of the smoking population are motivated to quit. ${ }^{6}$ Despite free smoking cessations services are readily available and cessation medication prescribed for free in these services, only about $2 \%$ of current smokers have used these aids. ${ }^{6}$ Majority of current smokers $(96.9 \%)$ claimed that they would not use these cessation aids to quit. ${ }^{6}$ Therefore, cessation aids are severely 
under-used. More effort in promoting use of cessation aids in quit attempts is needed.

Since the substantial expansion of smoke-free areas in Hong Kong in 2007, clustering of smokers at many outdoor smoking 'hotspots' such as the exits of railway stations, entrances of commercial buildings and shopping malls have been emerged. These smoking hotspots are non-smoke-free urban places, where many smokers gather to smoke around a rubbish bin with an ash tray to collect cigarette butts. We approached smokers at these 'hotspots' to promote smoking cessation and found this approach efficient in delivering brief advice to a larger number of smokers at low costs. ${ }^{78}$ However, brief advice had a small and non-significant effect on quitting outcomes, as most quit attempters still did not use cessation services or medications for quitting. ${ }^{9-11}$

We propose to deliver cessation aids such as nicotine replacement therapy (NRT) sample to further enhance their motivation to quit and take real action. NRT is a safe pharmacotherapy that replaces the nicotine usually provided by smoking cigarettes, to reduce craving and withdrawal symptoms ${ }^{12}$ and increase long-term abstinence. ${ }^{1314}$ In Hong Kong, NRT is included in the Hospital Authority Drug Formulary and can be purchased over-thecounter or obtained free-of-charge by enrolling in cessation clinics. In Hong Kong quit attempters, only 11.7\% had ever used prescribed or over-the-counter medication, and $14.2 \%$ used cessation services. ${ }^{6}$ The low prevalence of NRT usage may be explained by the high price of a pack of NRT compared with a pack of cigarettes, ${ }^{15}$ and smokers' low awareness and intention in using cessation services. $^{6}$

Providing NRT sample address the high cost to use NRT and encourage smokers to attempt quitting by using NRT. By trying NRT, smokers develop greater motivation and self-efficacy in quitting, ${ }^{16}$ which in turn promote quit attempts and prolong abstinence. ${ }^{17-19}$ Previous trials showed that NRT samples via mail, with additional behavioural supports such as telephone counselling, increased abstinence. ${ }^{17-20}$ We expected that delivering NRT sample is feasible in smoking hotspots recruitment, because the advice on using NRT is not complicated. We deem NRT sample effective for earlier quit attempts as they do not need to wait for a formal enrolment of cessation service and medication prescription, which may reduce their quitting intention. Also, earlier experience of using NRT help users identify the barriers and enhance counsellors to intervene in the later counselling session. Our previous pilot randomised controlled trial (RCT) showed that NRT sample substantially increased NRT use (34\% vs $2 \%$; adjusted risk ratio $(\mathrm{ARR})=15.13, \mathrm{p}<0.01)$, and insignificantly increased quit attempts (26\% vs $12 \%$; ARR $=1.51$, $\mathrm{p}=0.42$ ) at 3-month follow-up. ${ }^{21}$ We speculated that the limited intervention effect was due to the very brief medication advice onsite and lack of 'booster' follow-up. Therefore, to increase NRT adherence after the NRT delivery, we propose a nurse-led brief medication advice, onsite cessation service referrals and a booster follow-up.
This RCT aims to evaluate the effectiveness of delivering a 1-week NRT sample to adult smokers recruited at outdoor smoking hotspots for quit attempts (no smoking for at least 24hours) and use of smoking cessation services assessed at 1-month follow-up. Other quitting outcomes, including tobacco abstinence and self-efficacy and recruitment outcomes are also evaluated.

\section{METHODS AND ANALYSIS \\ Study design}

This study is a two-arm, pragmatic, multisite, cluster RCT. Trained outreach smoking cessation ambassadors and clinic staff will proactively approach and recruit the smokers at smoking hotspots in various districts in Hong Kong. The recruitment sessions will be randomised into the intervention and control group. Each recruitment session will last for about 3-4hours. The intervention group will receive 1-week free NRT sample, nurse-led medication advice and cessation service referral immediately after recruitment, while the control group will only receive the same advice and referral. We hypothesised that the recruitment sessions providing free NRT sample will recruit more smokers to the RCT, and the intervention group will achieve greater rate of quit attempt and cessation service use at 1-month follow-up. The study flow is shown in figure 1.

This RCT is coorganised by the smoking cessation research team of the University of Hong Kong (HKU) and the smoking cessation service of Tung Wah Group of Hospitals (TWGHs). As a pragmatic RCT, the recruitment procedures and intervention are specially designed so that these procedures will not interfere the smoking cessation services received from the TWGHs. For instance, participants' consent to participate in the RCT is sought after they received the intervention, and participants can refuse to participate in this RCT but still receive the nurse advice and service referral provided by the TWGHs.

All authors have no competing interest. This study is solely supported by the Food and Health Bureau of the Hong Kong Government. As this study has no support from tobacco or pharmaceutical companies, an independent data committee is not needed.

This study has no patient and public involvement in the study design, recruitment and result dissemination.

\section{Participants}

Smokers with the following inclusion criteria will be invited to participate in our RCT: (1) Hong Kong residents, (2) aged 18-65 years, (3) have used any tobacco products daily in the past month, (4) able to read and speak Chinese, (5) have not used NRT for the past month, (6) no severe angina, serious cardiac arrhythmias and hypertension, (7) have not suffered acute myocardial event in the past 4 weeks, (8) neither pregnant nor breast feeding, (9) not under medication and treatment due to mental illness. Criteria (6)-(9) are used to identify participants who are fit for using NRT in accordance with the 


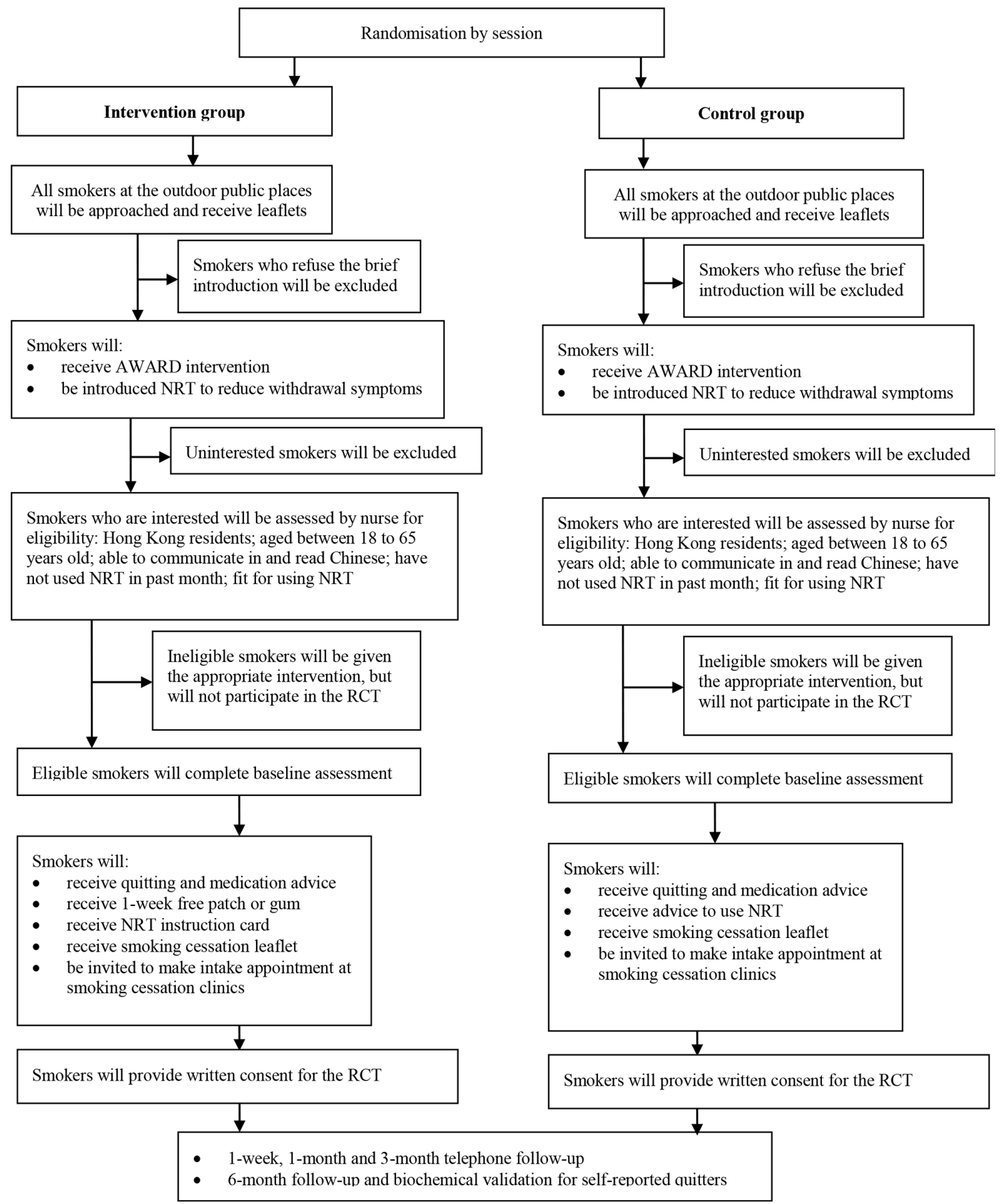

Figure 1 Recruitment and study flowchart. NRT, nicotine replacement therapy; RCT, randomised controlled trial.

local guideline for prescribing smoking cessation medication. ${ }^{22}$ Ineligible smokers can still receive our smoking cessation intervention, but they will not be included in the RCT.

The original study protocol (registered in October 2018) excluded smokers who consume less than 10 cigarettes per day in the RCT, as we intended to recruit smokers who have moderate to strong level of craving and a greater need for NRT. However, we considered that some participants have already reduced their cigarette consumption at study recruitment, but they still need NRT to relieve craving and withdrawal. The present study aims to promote the use of NRT for quit attempts with NRT sampling, so we decided to change this criterion to 'have used any tobacco products daily in the past month'. We revised this in the protocol and updated in the trial registry in March 2019.

\section{Recruitment procedures}

The recruitment staff will approach and distribute leaflets and pamphlets to the smokers at outdoor smoking hotspots, which promote smoking cessation and the services provided by TWGHs. If a smoker is willing to accept them and talk to the staff, the staff will advise the 
smoker to quit using the Ask, warn, advise, refer and do-itagain (AWARD) protocol. The AWARD protocol includes (1) ask the smoking history, (2) warn about the high risk (eg, half of the smokers will die of smoking-related diseases), (3) advise to quit, (4) refer to the smoking cessation clinics and (5) repeat the above advice (do-itagain). Our previous studies have shown that the AWARD protocol is a feasible and appropriate tool used by nonhealthcare professionals to promote smoking cessation. If the smoker is interested in smoking cessation, the staff will introduce NRT to the smoker and refer him/her to the onsite nurse. The intervention and assessment will mostly be taken place in a smoking cessation truck when the recruitment venues allow us to park the truck nearby, or at outdoor areas when a parking space for a truck is not available.

\section{Intervention}

In the intervention group, an onsite nurse will assess the eligibility of each participant. If the participant is willing to use NRT to quit smoking and meet all eligibility criteria, he/she will be prescribed 1-week NRT sample. The nurse will help the participant decide which type of NRT product (gum: $2 \mathrm{mg}$, patch: $14 \mathrm{mg}$ or $21 \mathrm{mg}$ ) that he/she can use and advise him/her on how to use the NRT based on his/her smoking habit and daily cigarette consumption. In addition, the nurse will deliver medication advice which addresses five main components: (1) the benefits for using NRT in quitting, (2) withdrawal symptoms due to smoking cessation, (3) side effects of NRT, (4) instructions for using NRT and (5) making appointments for smoking cessation clinics of TWGHs, which was adopted from the counselling protocol of our previous study (table 1 ). ${ }^{23}$ An instruction card of using NRT will be given. Participants will be advised to either stop or reduce smoking when using the NRT.

In the control sessions, the onsite nurse will perform similar assessment and deliver the medication advice, but they will not deliver an NRT sample and the NRT instruction card. Instead, they will advise the participants to obtain free NRT by enrolling in the smoking cessation clinics later. Both intervention and control groups will receive a one-page leaflet provided by the smoking cessation clinics, and encouraged to make service appointments with the smoking cessation clinics. The leaflet briefly introduced this RCT, the use of NRT and the services provided by the smoking cessation clinics.

After completing the above procedures, the research assistant or the nurse will introduce the RCT of evaluating the effect of NRT sample and the outreach service, and seek his/her written consent to participate. If the participant does not consent to participate in the RCT, he/she can still receive the smoking cessation intervention, but they will not be included in the RCT.

Some smokers are willing to receive the nurse-led intervention, but they have no time to receive all intervention or complete all recruitment procedures at the hotspots. If so, the recruitment staff will only briefly introduce the intervention and RCT, ask the participants to give written consent for participation. A nurse will contact these participants to complete the baseline questionnaire and deliver the intervention (medication advice and NRT prescription) via telephone within the same day. If the participants are in the intervention group, after the

Table 1 The five components of medication advice

Components Content

Withdrawal symptoms due to smoking cessation

Quitting smoking may cause craving, irritability, insomnia, frustration, anxiety, restlessness, etc

Benefits of using NRT in quitting

Nicotine gum and patch can help relieve these symptoms Scientific evidence strongly supports that NRT increases the quit rate and is a safe product

Side effects of NRT

Minor side effects may appear such as insomnia, skin irritation, jaw ache, hiccups and mouth soreness, but they will disappear after a few days

Instructions of using NRT

Patch: apply patch on clean and dry skin on the chest, back, upper arms, hips etc. Apply one patch per day. Change patch site daily to avoid skin irritation

Gum: steps of chewing gum: slowly chew the gum 10-15 times $\rightarrow$ the taste gradually becomes stronger $\rightarrow$ park the gum in the buccal area for about 1-2 min $\rightarrow$ the taste gradually becomes lighter $\rightarrow$ repeat the above steps. Avoid soft drinks, coffee and fruit juice $15 \mathrm{~min}$ before chewing. Daily dosage should not be more than 15 pieces is allowed

NRT, nicotine replacement therapy. 
telephone counselling by the nurse, our research staff will send the NRT sample via mail within 2 days.

\section{Follow-up}

All participants will be contacted via telephone by a nurse or a trained research staff 1 week after recruitment. The aim of this follow-up is (1) to enquire if there is any progress towards smoking cessation and (2) encourage the use of NRT for a quit attempt and/or enrolment in the smoking cessation clinics as soon as possible. If participants show no interest to quit, the nurse/research staff will use the ' $5 \mathrm{R}$ ' approach to remotivate the quitting intention. The ' $5 \mathrm{R}$ ' approach is being adopted by the local and international smoking cessation guideline including the discussion on why quitting is important (relevance), hazards of smoking (risk), benefits of quitting (rewards), difficulties (roadblocks) and repeated quit attempts (repetition). If participants report any contra-indication(s) to NRT, the nurse/research staff will discuss the reasons with them, and, if necessary, refer the participants to the physician in the smoking cessation clinic. If the participant loses the follow-up, we will send a WhatsApp message to him and invite him for a discussion about his/her quitting. Some participants will book an appointment to enrol the standard smoking cessation service in the smoking cessation clinics within 2 weeks of the baseline recruitment, and hence the 1-week follow-up will not be needed.

\section{Randomisation}

We will use cluster randomisation based on promotion session as the cluster unit because the procedures of individual randomisation are difficult in outdoor areas as found in our pilot RCT. This cluster randomisation also prevents participants from hearing the intervention content of another arm. The principal investigator has generated multiple lists of group allocation, which are based on random numbers from a computer programme of random number generator. Each list corresponds to a recruitment place. Each month the staff of TWGHs will generate a list of 15-25 places that the recruitment sessions will take place in the next month. The recruitment staff of HKU research team will randomly allocate each promotion session to either trial arm following the lists generated by the principal investigator.

\section{Allocation concealment}

All recruitment staff and the principal investigator will know the group allocation before each promotion session, so there will be no group concealment.

\section{Blinding}

Participants, recruitment staff and nurses cannot be blinded to the intervention. Assessors of the follow-up outcomes and the research investigators will not be involved in the recruitment and intervention delivery, so they will be blinded to the group allocation (single blindness).

\section{Baseline assessments}

Baseline information includes sociodemographics, current use of conventional and new tobacco products, such as heat-not-burn tobacco products and electronic cigarettes, history of quit attempts and intention to quit. Nicotine dependence will be assessed by the Fagerström Test for Nicotine Dependence. ${ }^{24}$ Participants will also be asked about their self-efficacy in quitting, based on the perceived importance, difficulty and confidence to quit smoking on a Likert scale from 0 to $10 .^{25}$

\section{Outcomes}

At 1-month, 3-month and 6-month follow-up, the quitting outcomes will be assessed by trained interviewers through telephone who are blinded to the subject's group allocation. A small amount (HK\$50=US\$6.4) of cash voucher will be offered to those who completed a follow-up survey. At 6-month follow-up, participants who reported no smoking in past 7 days will be invited for a biochemical validation of the abstinence. Participants with exhaled carbon monoxide $4 \mathrm{ppm}$ or below and saliva cotinine level below $10 \mathrm{ng} / \mathrm{mL}$ will be considered as validated abstinence. ${ }^{26}{ }^{27}$ Participants of the biochemical validation, no matter the abstinence status is confirmed, will be offered an HK\$50 (US\$6.4) cash voucher for the travel compensation. The two primary outcomes are the proportion of smokers who enrol in any cessation service in Hong Kong within 1 month of the recruitment, and the proportion of smokers who report quit attempts at 1-month follow-up. Secondary outcomes include self-reported use of NRT at 1-month and 3-month, self-reported 7-day tobacco abstinence at 3 months and 6 months, 30-day abstinence at 3 months and 6 months, biochemically validated abstinence at 6 months, perceived importance, difficulty and confidence to quit (scale 0-10), and Incremental Behavior Change towards Smoking Cessation. ${ }^{28}$

Process outcomes include number of smokers who will be approached, accept the brief smoking cessation advice or be recruited to participate in the RCT. All these indicators will be documented by the research assistants in the recruitment sessions.

\section{Sample size determination}

As we predicted that the recruitment sessions of providing free NRT samples will recruit more smokers, participant ratio of the two trial groups is arbitrarily set at 2:1. At the time we designed this trial, our preliminary analysis of the aforementioned pilot RCT showed that the rate of quit attempt in the intervention and control group was $26 \%$ and $12 \%$ at 3-month follow-up, respectively. We assumed that quit attempt rate at 1 month should be lower than that measured at 3 months, when our questions are about any attempts since participating in the trial. Hence, we arbitrarily reduced these rates to $20 \%$ and $8 \%$ for 1-month follow-up. To detect a significant difference with normal test and a power of $95 \%$ and $5 \%$ significance level, we need 485 subjects in the RCT (allocation ratio $2: 1 ; 323$ vs 162). Based on the method of Eldridge $e t a l^{29}$ 
conservatively assuming that we could recruit eight participants per session on average and the intracluster correlation coefficient was 0.1 , the design effect is estimated to be 1.7. Thus, the minimum sample size required for the trial is $825(=1.7 \times 485)$ participants and 60 sessions in total. With the similar calculation, the required sample size for detecting a significant difference in the proportion of using any smoking cessation service is 421 .

\section{Statistical analysis}

An independent data analyst will perform the data check of missing, duplication and validity on the completed dataset before analysis is done. All data will be de-identified in the data analysis. To assess the effect of NRT sampling to the process outcomes, the number of smokers who accept the brief advice or consent the RCT in each recruitment session will be the outcome variables of Poisson regression model, with group allocation as the predictor and total number of approached smokers in that promotion session as the offset variable. Considering the possibility of correlated outcomes of participants within the recruitment session, an analysis by generalised estimating equations model, assigning session as a random effect, will be used to summarise the intervention effect on the primary outcome. The analysis adopted a longitudinal approach with an exchangeable structure for the correlation matrix of the outcome. Other secondary outcomes will be analysed with either generalised estimating equations models (binary outcomes; eg, tobacco abstinence) or linear mixed model (continuous outcomes; eg, perceived importance to quit smoking). Both intention-to-treat (treating participants as smokers without quit attempts and no use of cessation services if they are lost to follow-up) and per protocol analysis will be done. Subgroup analysis of comparing the primary and secondary outcomes between participants receiving the nurse-led intervention onsite and those receiving the intervention via telephone will be done.

\section{Ethics and dissemination}

Some NRT users may experience a local skin reaction. The reaction is usually mild and self-limiting, but occasionally worsens over the course of therapy. In fewer than $5 \%$ of patients, such reaction requires the discontinuation of the NRT. Other side effects are insomnia and/or vivid dreams. Common side effects of nicotine gum include mouth soreness, hiccups, dyspepsia and jaw ache. These effects are generally mild and transient, and can often be alleviated by correcting the patient's chewing technique or correcting the patient's using habit. All participants will be asked at 1-week telephone follow-up if they suffer from these side effects. If the side effects are serious, the follow-up staff will ask the participants to cease usage and receive further advice from the doctors in the next clinic appointment.

The present study has already been approved by the Institutional Review Board of the University of Hong Kong/Hospital Authority Hong Kong West Cluster approved the trial (UW 18-118). Findings will be disseminated through funding website, publication and conference presentations. De-identified datasets are available on request made to the corresponding author.

\section{DISCUSSION}

The RCT described in this protocol has several significant implications. First, unlike conventional smoking cessation services which aim at smokers who are motivated to quit with smoking cessation aids, our target participants include smokers who have or have not used any cessation aids. Previous population survey has shown that majority of quitters do not initiate to use cessation services or medications for quit attempts. ${ }^{6}$ In Hong Kong, NRT is an over-the-counter medication and can be bought from pharmacy, yet at a relatively high price. ${ }^{15}$ Although smokers can obtain free NRT by enrolling in smoking cessation clinics, many of them are either unaware or unwilling to seek cessation services. ${ }^{6}$ Therefore, it is of great importance to outreach, and develop new strategies of motivating the smokers to use these aids. Second, we adopt a proactive approach to recruit and provide immediate assistance to smokers at smoking hotspots when they are recruited. We believe that these smokers are probably 'behaved' smokers, who choose not to smoke indoor, and may accept our outreach promotion. Our experiences supported this, ${ }^{8}$ and further showed that the outreach promotions enable us to reach our target participants who have intention yet no action to quit smoking. ${ }^{3031}$ Our goal is to raise these smokers' motivation by delivering brief advice and 1-week free NRT sample. If the intervention is found useful, the trial will show that accessible and immediate interventions delivered at smoking hotspots can improve recruitment and cessation outcomes. Finally, the present RCT aims to evaluate the effectiveness of 'delivering' NRT sample, but the findings cannot ascertain the efficacy of the 1-week NRT. Participants are prescribed NRT sample but their complete NRT compliance is not mandatory, and participants can freely choose whenever to start or stop using NRT. Hence, the detected benefits on smoking cessation in this RCT, if any, can be explained by the study intervention. This possible finding will be more relevant to support decisions on delivering the intervention.

The present RCT has three limitations. First, the present RCT will use a cluster RCT design, in which recruitment sessions are randomised to either trial arm before each session, hence we cannot conceal group allocation of the recruitment staff. Also, study outcomes from participants within the same recruitment session may be correlated. In spite of these disadvantages, cluster RCT design applied in this outdoor recruitment can simplify and shorten recruitment procedure, and prevent participants from hearing the intervention content of another arm. Our previous study showed that cluster randomisation of smoking patients at outdoor smoking hotspots did not lead to imbalance of baseline characteristics. ${ }^{9}$ 
Generalised estimation equation model can be used to analyse outcomes which may be correlated within clusters. Second, as a trial of inducing participation and motivating quitting intention, we allow some flexibilities in recruitment and intervention delivery. For instance, participants could choose to receive the medication advice via a subsequent telephone follow-up if they have no sufficient time onsite. We do not restrain the recruitment staff from delivering intervention content if participants have enquires about smoking cessation and NRT use, before they consent to participate in the RCT. These practices may lead to intervention delivery before they consent to participate in the RCT and baseline assessment. Nonetheless, all recruitment procedures and intervention contents will be documented for later analysis and considerations. Finally, as the onsite nurses and recruitment staff will help the participants to book appointment in the cessation clinics, participants in this study will be more likely to use smoking cessation services than those in our previous pilot RCT. Hence, the effect size due to the delivery of NRT sample may be reduced, especially in the subgroup who use the cessation services. Findings may not be fully applicable to other intervention designs that do not refer cessation services.

\section{CURRENT STATUS}

Recruitment started on 13 October 2018. All outreach activities, follow-up and data collection will be expected to be completed July 2020. The protocol was amended in March 2019. The amendment was to allow participants who smoked less than 10 cigarettes a day to participate in the RCT. The research questions, outcomes and data analysis method remained unchanged throughout the study period.

\section{Human rights}

All procedures performed in this study involving human participants were in accordance with the ethical standards of the institutional and/or national research committee and with the 1964 Declaration of Helsinki and its later amendments or comparable ethical standards. The trial was approved by the Institutional Review Board of the University of Hong Kong/Hospital Authority Hong Kong West Cluster (IRB: UW 18-118).

\section{Author affiliations}

${ }^{1}$ School of Nursing, The University of Hong Kong, Hong Kong, Hong Kong ${ }^{2}$ Integrated Centre on Smoking Cessation, Tung Wah Group of Hospitals, Hong Kong, Hong Kong

${ }^{3}$ School of Public Health, The University of Hong Kong, Hong Kong, Hong Kong

Acknowledgements On behalf of the project team, we express our deep appreciation to all the smoking cessation clinics under Tung Wah Group of Hospitals for facilitating all recruitment activities. We are grateful to all the project coordinators, including Ms Tiffany Lai, Ms Chloe Lau, Mr Ken Lin, Mr Andy Ho, Ms Anna Lau and Mr Timmy Man, for their effort to recruit participants and administrative duties.

Contributors The corresponding author confirms that all listed authors meet authorship criteria and that no others meeting the criteria have been omitted.
YTDC and CWHL were responsible for literature search, data analysis, data interpretation, and manuscript writing. YTDC, CHHC, KSH, CT, WHCL, MPW and THL were responsible for the staff training and intervention design. CT and CWHL were responsible for fieldwork supervision, data collection. All authors reviewed the manuscript and approved for submission.

Funding This work was supported by Health Care and Promotion Scheme of the Health Medical Research Fund, Food and Health Bureau, the Hong Kong SAR Government (grant number 01170418). The funder has no roles in study design, collection, management, analysis and interpretation of data.

Competing interests THL is the principal investigator of two-family well-being projects, and MPW is the coinvestigator of one of the two projects funded by Hong Kong Jockey Club Charities Trust. All other authors have no connection with tobacco, alcohol, pharmaceutical or gaming industries or anybody substantially funded by these organisations.

Patient and public involvement Patients and/or the public were not involved in the design, or conduct, or reporting, or dissemination plans of this research.

\section{Patient consent for publication Not required}

Provenance and peer review Not commissioned; externally peer reviewed.

Open access This is an open access article distributed in accordance with the Creative Commons Attribution Non Commercial (CC BY-NC 4.0) license, which permits others to distribute, remix, adapt, build upon this work non-commercially, and license their derivative works on different terms, provided the original work is properly cited, appropriate credit is given, any changes made indicated, and the use is non-commercial. See: http://creativecommons.org/licenses/by-nc/4.0/.

\section{ORCID iDs}

Yee Tak Derek Cheung http://orcid.org/0000-0002-5850-5349

William Ho Cheung Li http://orcid.org/0000-0002-3195-7695

Man Ping Wang http://orcid.org/0000-0003-4000-2388

\section{REFERENCES}

1 Census \& Statistics Department (Hong Kong SAR government). Thematic household survey, report No. 59: pattern of smoking. Hong Kong: Census \& Statistics Department, 2016.

2 Lam TH. Absolute risk of tobacco deaths: one in two smokers will be killed by smoking. Arch Intern Med 2012;172:845-6.

3 World Health Organization. Global progress report on implementation of the who framework convention on tobacco control. 2012. Geneva: World Health Organization, 2012.

4 ITC Project, China Chinese Center for Disease Control and Prevention (CDC) Tobacco Control Office. Itc China executive summary report. findings from the wave 1 to 5 surveys (2006-2015. Beijing, China: University of Waterloo, Canada and Tobacco Control Office, CDC, 2017.

5 Wang L, Jin Y, Lu B, et al. A Cross-Country study of smoking cessation assistance utilization in 16 low and middle income countries: data from the global adult tobacco survey (2008-2012). Nicotine Tob Res 2016;18:1076-82.

6 Census \& Statistics Department (Hong Kong SAR government). Thematic household survey, report No. 64: pattern of smoking. Hong Kong: Census \& Statistics Department, 2018.

7 Chan SSC, Cheung YTD, Wan Z, et al. Proactive and Brief Smoking Cessation Intervention for Smokers at Outdoor Smoking "Hotspots" in Hong Kong. J Canc Educ 2018;33:365-70.

8 Cheung YTD, Lam TH, Li WHC, et al. Feasibility, Efficacy, and Cost Analysis of Promoting Smoking Cessation at Outdoor Smoking "Hotspots": A Pre-Post Study. Nicotine Tob Res 2018;20:1519-24.

9 Chan SSC, Cheung YTD, Wong YMB, et al. A brief smoking cessation advice by youth counselors for the smokers in the Hong Kong quit to WIN contest 2010: a cluster randomized controlled trial. Prev Sci 2018;19:209-19.

10 Chan SSC, Wong DCN, Cheung YTD, et al. A block randomized controlled trial of a brief smoking cessation counselling and advice through short message service on participants who joined the quit to WIN contest in Hong Kong. Health Educ Res 2015;30:609-21.

11 Cheung YTD, Wang MP, Li HCW, et al. Effectiveness of a small cash incentive on abstinence and use of cessation AIDS for adult smokers: a randomized controlled trial. Addict Behav 2017;66:17-25.

12 West R, Shiffman S. Effect of oral nicotine dosing forms on cigarette withdrawal symptoms and craving: a systematic review. Psychopharmacology 2001;155:115-22. 
13 Kasza KA, Hyland AJ, Borland R, et al. Effectiveness of stopsmoking medications: findings from the International tobacco control (ITC) four country survey. Addiction 2013;108:193-202.

14 West R, Raw M, McNeill A, et al. Health-Care interventions to promote and assist tobacco cessation: a review of efficacy, effectiveness and affordability for use in national Guideline development. Addiction 2015;110:1388-403.

15 Abdullah AS, Hedley AJ, Chan SSC, et al. A randomized controlled trial of two different lengths of nicotine replacement therapy for smoking cessation. Biomed Res Int 2013;2013:9

16 Burris JL, Heckman BW, Mathew AR, et al. A mechanistic test of nicotine replacement therapy sampling for smoking cessation induction. Psychol Addict Behav 2015;29:392-9.

17 Jardin BF, Cropsey KL, Wahlquist AE, et al. Evaluating the effect of access to free medication to quit smoking: a clinical trial testing the role of motivation. Nicotine Tob Res 2014;16:992-9.

18 Carpenter MJ, Hughes JR, Gray KM, et al. Nicotine therapy sampling to induce quit attempts among smokers unmotivated to quit: a randomized clinical trial. Arch Intern Med 2011;171:1901-7.

19 Bush TM, McAfee T, Deprey M, et al. The impact of a free nicotine patch starter kit on quit rates in a state quit line. Nicotine Tob Res 2008;10:1511-6.

20 Dahne J, Wahlquist AE, Boatright AS, et al. Nicotine replacement therapy sampling via primary care: methods from a pragmatic cluster randomized clinical trial. Contemp Clin Trials 2018;72:1-7.

21 Cheung YTD, Li HCW, Wang MP, et al. Delivery of a nicotine replacement therapy sample at outdoor smoking hotspots for promoting quit attempts: a pilot randomized controlled trial. Nicotine Tob Res 2019. doi:10.1093/ntr/ntz138. [Epub ahead of print: 12 Aug 2019].

22 Tobacco Control Office DoH, Hong Kong SAR government. Smoking cessation information kit Hong Kong: department of health, Hong Kong SAR government; 2015, 2017. Available: https:// www.tco.gov.hk/english/quitting/files/kit15.pdf [Accessed 25 July 2017].

23 Cheung YTD, Leung JPK, Cheung CKC, et al. Motivating smokers at outdoor public smoking hotspots to have a quit attempt with a nicotine replacement therapy sample: study protocol for a randomized controlled trial. Trials 2016;17:1-8.

24 Heatherton TF, Kozlowski LT, Frecker RC, et al. The Fagerström test for nicotine dependence: a revision of the Fagerström tolerance questionnaire. Br J Addict 1991;86:1119-27.

25 Wang MP, Suen YN, Li WH-C, et al. Intervention with brief cessation advice plus active referral for proactively recruited community smokers: a pragmatic cluster randomized clinical trial. JAMA Intern Med 2017;177:1790-7.

26 Cropsey KL, Trent LR, Clark CB, et al. How low should you go? determining the optimal cutoff for exhaled carbon monoxide to confirm smoking abstinence when using cotinine as reference. Nicotine Tob Res 2014;16:1348-55.

27 Montalto NJ, Wells WO. Validation of self-reported smoking status using saliva cotinine: a rapid semiquantitative dipstick method. Cancer Epidemiol Biomarkers Prev 2007;16:1858-62.

28 Flocke SA, Step MM, Lawson PJ, et al. Development of a measure of incremental behavior change toward smoking cessation. Nicotine Tob Res 2016;20:73-80.

29 Eldridge SM, Ashby D, Kerry S. Sample size for cluster randomized trials: effect of coefficient of variation of cluster size and analysis method. Int J Epidemiol 2006;35:1292-300.

30 Cheung YTD, Lam TH, WHC L, et al. Feasibility, Efficacy, and Cost Analysis of Promoting Smoking Cessation at Outdoor Smoking "Hotspots": A Pre-Post Study. Nicotine \& Tobacco Research2017:ntx 147-ntx47.

31 Chan SSC, Cheung YTD, Wan Z, et al. Proactive and Brief Smoking Cessation Intervention for Smokers at Outdoor Smoking "Hotspots" in Hong Kong. J Cancer Educ 2018;33:365-70. 